\title{
Influence of pyramid size on reflectivity of silicon surfaces textured using an alkaline etchant
}

\author{
AMMAR MAHMOUD AL-HUSSEINI* and BASHAR LAHLOUH \\ Department of Physics, University of Jordan, Amman 11942, Jordan \\ *Author for correspondence (a.taha@ju.edu.jo)
}

MS received 27 September 2018; accepted 11 January 2019; published online 13 May 2019

\begin{abstract}
Surface texturing of p-type monocrystalline silicon (100) is well known as one of the best methods to reduce reflection losses and to increase light trapping and light absorption probability. Pyramid surface textures play a major role in reducing the reflectance of monocrystalline silicon surfaces. In this paper, the size of pyramids formed on the surface of p-type silicon substrates and by changing the etching characteristics during the texturing process of silicon were studied and evaluated. The pyramids that formed on the crystalline silicon surface formed light traps that led to increased light absorption efficiency. The pyramid size effects on the percent reflectivity were evaluated at normal incidence and an inverse relationship between the percent reflectivity and the pyramid size was found. The size of the pyramids was controlled by controlling the texturing process by changing the concentrations of potassium hydroxide (KOH) and isopropyl alcohol (IPA) and by controlling the etching process time. In this work, the optimized etching conditions were determined as a solution prepared with $20 \mathrm{wt} \% \mathrm{KOH}$ and $3 \mathrm{wt} \%$ IPA for wet etching at a reaction temperature of $80^{\circ} \mathrm{C}$ and an etching time of $40 \mathrm{~min}$. The lowest value for percent reflectivity of the patterned surfaces was $9.7 \%$ and it was achieved for pyramid bases close to $4 \mu \mathrm{m}$ as measured at a wavelength of $650 \mathrm{~nm}$.
\end{abstract}

Keywords. Pyramid structure; surface morphology; light trapping; reflectivity; spectral reflectivity; surface modification.

\section{Introduction}

Silicon substrates with various surface structures of different shapes and sizes are widely used in many research and industrial applications. Patterned silicon is popular and widely used, for example, to improve the efficiency of solar cells by increasing light trapping and minimizing light reflectivity of the textured silicon surfaces [1-3], for device fabrication of microelectromechanical system [4-6] and in many biological applications $[7,8]$. Since light surface reflections are among the major loss mechanisms in materials, different surface modifications were attempted by many research groups to control these losses [9]. Patterning of the silicon surface increases its surface topographical irregularities by increasing the surface area. This usually enhances the optical response of such surfaces. Thus, it increases its efficiency in converting light into an electric current [10]. Anisotropic etching of silicon properties has a promising role in manufacturing more efficient silicon solar cells by reducing the front surface reflection losses [11-13]. Figure 1 shows a schematic representation of a patterned silicon wafers with a front pyramid pattern and a flat back side. The incoming light ray has two chances to interact with the patterned Si surface. The first possibility is due to the reflection of the incident ray onto a neighbouring facet. The second possibility refers to the ray transmitted into the bulk of the Si substrate, the absorbing medium in this case; the transmitted ray is first reflected from the rear plane of the substrate and then, it is reflected again through the bulk of the Si substrate by the sides of the pyramids on the front side of the substrate $[14,15]$.

Light trapping due to multiple reflection and transmission increases when light is reflected from one facet to neighbouring facets. Light trapping increases since the transmitted light is at an angle to the perpendicular. This, in fact, leads to a longer first pass through the absorbing medium (1) and this also leads to internal reflections from the back surface of the absorbing medium (2). A patterned front surface also paves the way to longer internal reflections at the front surface (3). It can be noted here that when the transmitted light is at an angle perpendicular to the facets, more light is absorbed near the front surface; this can be an advantage or a disadvantage based on the location of the active material layers and the relative rates of interactions within the material itself [15].

Etching is usually used to form a pyramidal structure that can improve light collection and light trapping within monocrystalline silicon solar cells through internal reflections. Anisotropic wet etching, using potassium hydroxide $(\mathrm{KOH})$, is the most commonly used inorganic silicon etchant. Although other etching agents are successful in achieving better and smoother patterns, such as the ammonium tetraethyl hydroxide organic compound, but these etching compounds are much more expensive and are also known 


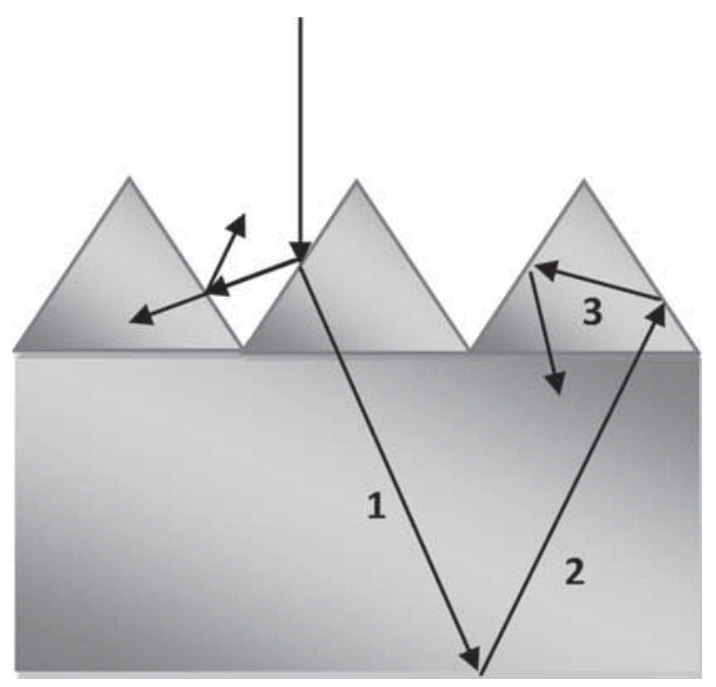

Figure 1. Light trapping and interactions with the pyramid structure.

mostly for their neurotoxicity, which poses significant health risks and hence require more appropriate safety measures [16-19].

In this study, the silicon surface structures based on free alkali profiling were studied by mixing $\mathrm{KOH}$, isopropyl alcohol (IPA) and distilled water. In this etching solution, IPA can help not only in removing hydrogen bubbles, but also in promoting the formation of large pyramids. Without IPA, the $\mathrm{KOH}$ solution cannot generate full surface pyramid coverage [20-23]. The silicon electrolyte energy should be reduced to achieve sufficient surface wetting for the silicon surface, which in turn can enhance the pyramid nucleation. Mixing active surface additives in the solution reduce interstitial energy [24]. Increasing the concentration of the IPA improves Si surface wetting, but the rate of silicon removal decreases further with increased IPA concentration [17]. Nonuniformities in the pyramid sizes or shapes were observed in wafers textured in a caustic solution [25]. Therefore, for the sake of cost reduction of solar cells, for better cell performance and for a more uniform pyramid size distribution, a careful design of the etching process has to be developed and evaluated [26]. The ultimate aim of this research work is to improve the surface morphology to achieve the lowest possible reflection by controlling the characteristics of the pyramid pattern on the surface of the etched silicon substrate. The pyramid shape, construction and size are all crucial factors for the surface interactions with the incoming light $[21,27]$. Optimal control of the structure of the etched pyramids requires accurate design of the different texturization conditions, such as time of etching and the concentration of the etching solution. This work presents an inexpensive and large-scale chemical process for the surface patterning of silicon surfaces for solar cell applications.

\section{Experimental}

Monocrystalline polished p-type (100) silicon wafers with a wafer thickness of $675 \pm 25 \mu \mathrm{m}$ and a resistivity of 3-30 $\Omega \mathrm{cm}$ were used as a starting surface. The silicon wafers were thoroughly cleaned using an acetone bath followed by an ethanol bath and, as a final step, the substrates were cleaned using distilled water. Silicon wafers were then immersed in $25 \%$ sodium hydroxide solution and the cleaning cycle mentioned above, was used again for these treated surfaces.

To prepare the pyramid patterns on the cleaned silicon surfaces, different concentrations of $\mathrm{KOH}$ and IPA solutions were prepared. Aqueous solution of $\mathrm{KOH}$ with concentrations ranging from $20 \pm 0.14$ to $28 \pm 0.14 \mathrm{wt} \%$ and IPA solutions with concentrations from $3 \pm 0.1$ to $9 \pm 0.1 \mathrm{wt} \%$ were used at $80 \pm 0.1{ }^{\circ} \mathrm{C}$ and the etching time was varied between 15 and $30 \mathrm{~min}$. The details of the etching process can be found in a previous work [27]. Different pyramid sizes were accomplished by changing the chemical concentrations and the etching time. This resulted in silicon surfaces with small, medium, big and mixed pyramid sizes and with complete or partial surface coverage of the patterned silicon surfaces. The surface patterns were studied using the field emission ionization scanning electron microscope (SEM) and the percent reflectivity of the patterned surfaces was measured using the FilmTek 3000 spectrophotometer in the wavelength range of 240-840 nm. Percent reflectivity measurements were carried out only at normal incidence. From the SEM images, two parameters were evaluated, namely the average pyramid size and the pyramid number density. The open source software, Imagej, was used to analyse the SEM micrographs [14]. This software can identify the tips of the pyramids and give a number density of pyramids in the SEM images. The results of these measurements were used to calculate the heights of the pyramids (h). The height calculation can easily be made in two dimensions, taking into consideration the geometry, as

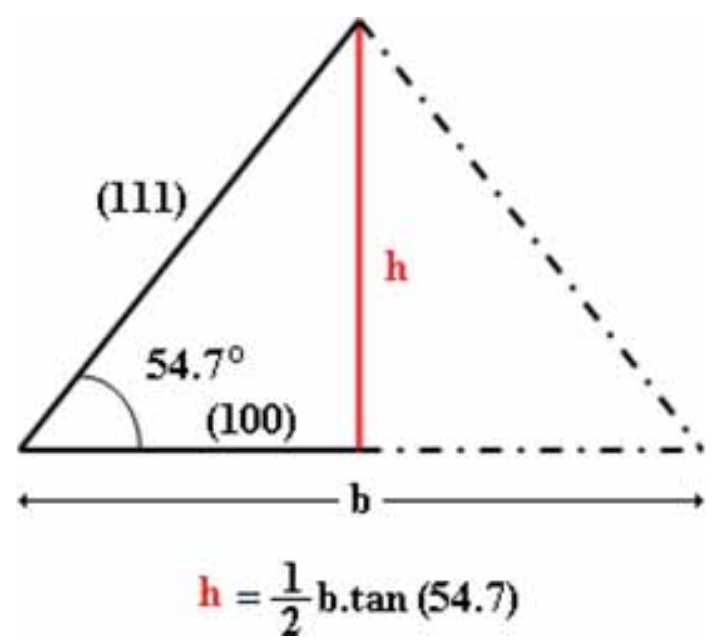

Figure 2. Representation of a pyramid on a textured mono-Siwafer in two dimensions. 
shown in figure 2. Using three SEM images for each etching condition, the mean and standard deviation of the pyramid sizes was calculated. The percent reflectivity measurements obtained using FilmTek 3000 were used to determine and compare the reflectivity values at the wavelength of $650 \mathrm{~nm}$.

\section{Results and discussion}

In the first set of samples, the IPA concentration was changed from 3 to $9 \mathrm{wt} \%$, while the $\mathrm{KOH}$ concentration was maintained at $20 \mathrm{wt} \%$, and the etching time was set for $40 \mathrm{~min}$ at $80^{\circ} \mathrm{C}$. Figure 3 shows the SEM images and the resulting morphologies of four samples at different concentrations of IPA. A careful inspection of figure 3 indicates that at $3 \mathrm{wt} \%$ IPA concentration, the pyramids completely cover the entire surface. As the IPA concentration is increased from 3 to 9 $\mathrm{wt} \%$, a rapid etching reaction rate is expected. The density of the formed pyramids is then limited by this etching reaction rate. The high reaction rates gradually reduce the density of pyramids covering the surface and lead to the breaking of the hydrogen bonding network by the collected alcohol. The rapid reaction rate leads to the formation of a near surface layer that hinders the formation of any more pyramids [14,28].

The effects of $\mathrm{KOH}$ concentration on the etching process were also studied. $\mathrm{KOH}$ solutions with concentrations of 20 , 24,26 and $28 \mathrm{wt} \%$, with the addition of $3 \mathrm{wt} \%$ of IPA solution at $80^{\circ} \mathrm{C}$ and for an etching time of $40 \mathrm{~min}$, were prepared. The SEM micrographs of the studied patterned substrates are shown in figure 4 . It is obvious that the shape and the surface coverage of the pyramids depend on the concentration of the $\mathrm{KOH}$ solution. At a $\mathrm{KOH}$ solution concentration of $20 \mathrm{wt} \%$, the pyramid size is large and the surface coverage of these pyramids is more uniform, but with increased concentration of $\mathrm{KOH}$, the etching rate decreased and the pyramids became smaller and did not fully fill the substrate area. The observed differences in the etched pyramid patterns can be explained as follows: as the $\mathrm{KOH}$ concentration is increased, the dissolution kinetics are altered such that the removal of silicon atoms from the crystalline surface is very high. This hinders the $\mathrm{Si}(\mathrm{OH})_{4}$ formation which cannot keep up with the high $\mathrm{Si}$ dissolution rate. The concentrations of these complexes are also increasing, and a protective layer forms near the silicon surface which prevents the etchants from accessing the silicon substrate surface [14].
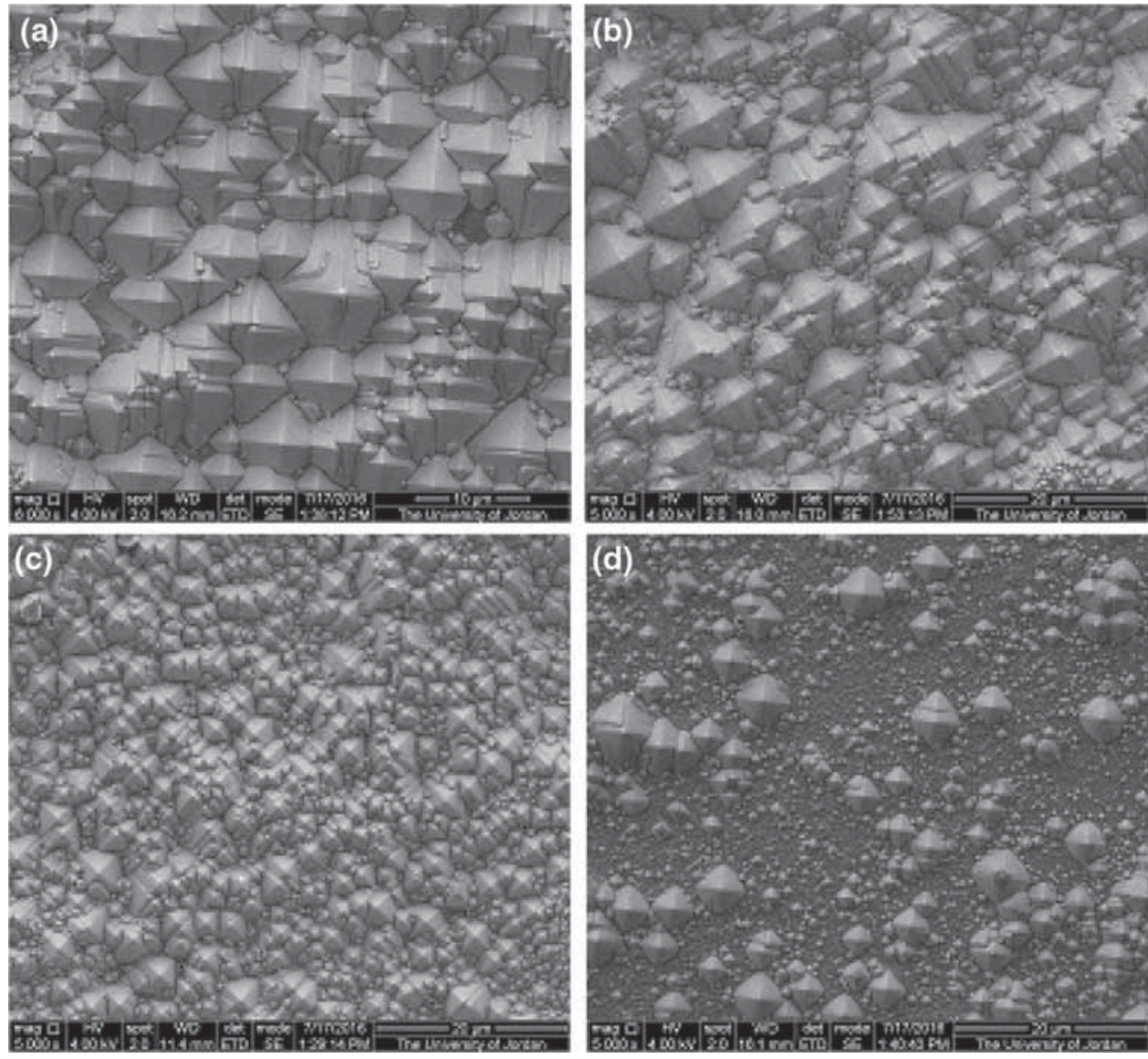

Figure 3. SEM micrographs for silicon substrates after etching with $20 \mathrm{wt} \% \mathrm{KOH}+$ IPA for $40 \mathrm{~min}$ at $80^{\circ} \mathrm{C}$ : (a) 3 , (b) 5 , (c) 7 and (d) 9 wt $\%$ IPA. 

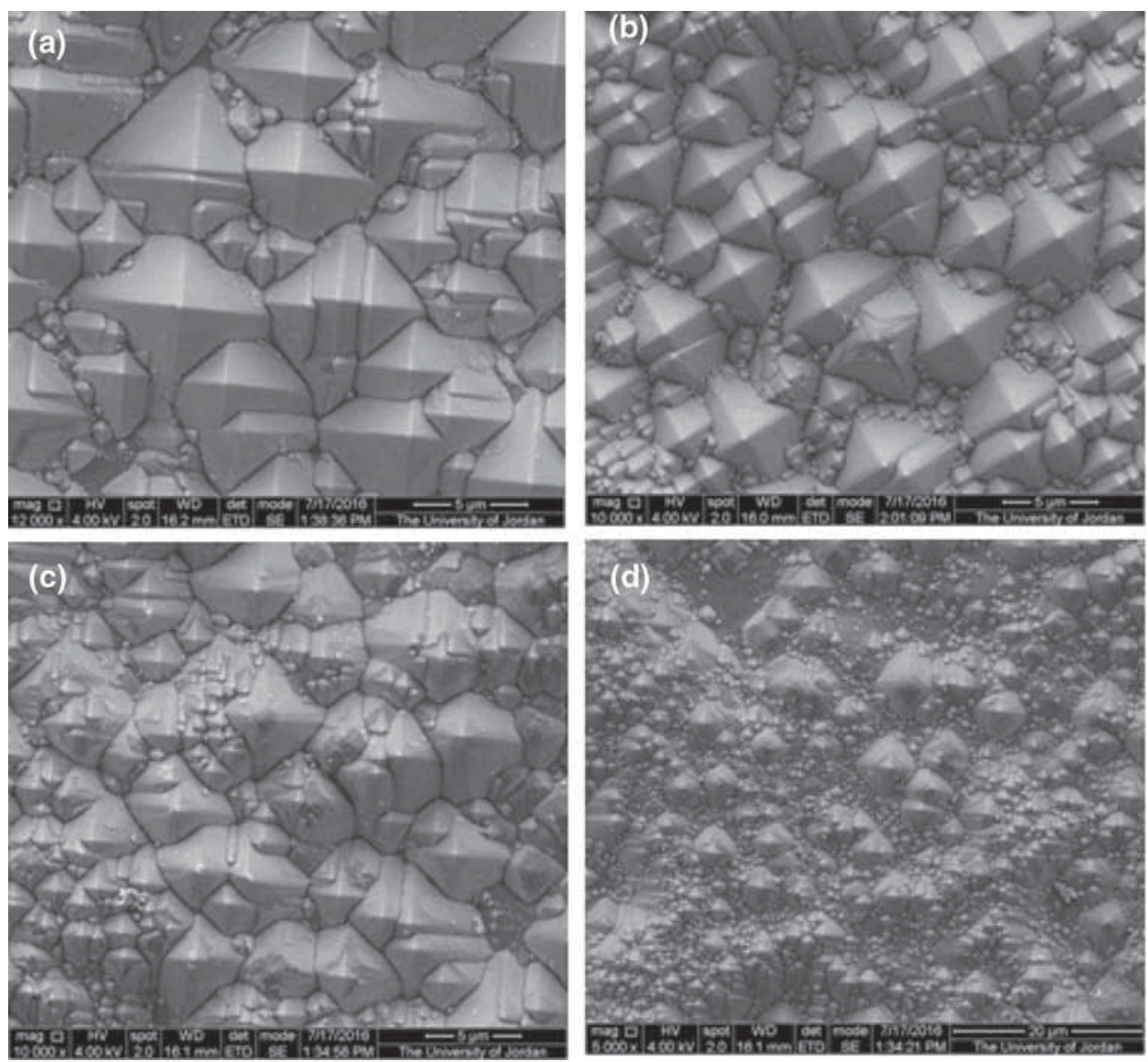

Figure 4. SEM images of the silicon surfaces after etching with different $\mathrm{KOH}$ concentrations +3 wt $\%$ IPA solution at $80^{\circ} \mathrm{C}$ for $40 \mathrm{~min}$ : (a) 20 , (b) 24 , (c) 26 and (d) $28 \mathrm{wt} \% \mathrm{KOH}$.

Etching time effects on silicon surface etching are also evaluated. Figure 5 shows the SEM images of textured monocrystalline $\mathrm{Si}$ wafers with a solution consisting of $20 \mathrm{wt} \% \mathrm{KOH}$ concentration and $3 \mathrm{wt} \%$ IPA for 15, 20, 25 and $30 \mathrm{~min}$ at $80^{\circ} \mathrm{C}$. At an etching time of $15 \mathrm{~min}$, small pyramid patterns distributed in patches with smooth areas between these patches are observed. The smooth areas are the regions vacant of any pyramid structures. This observation can be attributed to the relatively short etching time as shown in figure 5a. As the etching time is increased to $30 \mathrm{~min}$, the entire surface of the treated substrate was covered with small pyramids with some patches of larger pyramids. The pyramid size distribution is now more uniform as shown in figure $5 \mathrm{~d}$. The pyramids grew in size with an increase in etching time. By carefully examining figure $5 \mathrm{~b}$ and $\mathrm{c}$, one can see that the longer the etching time, the more uniform is the pyramid pattern.

The effects of surface patterning conditions on the specular surface reflectivity at normal incidence are measured and evaluated. The behaviour of silicon's surface percent reflectivity as a function of IPA concentration is presented in figure 6 . The IPA concentration was changed from 3 to $9 \mathrm{wt} \%$, while the $\mathrm{KOH}$ concentration was fixed at $20 \mathrm{wt} \%$, and the etching time was set for $40 \mathrm{~min}$ at $80^{\circ} \mathrm{C}$.
The percent reflectivity at normal incidence dropped over the whole studied wavelength range of $240-840 \mathrm{~nm}$ as the IPA concentration increased from 3 to $9 \%$. This can be attributed to the pyramid surface coverage and the pyramid size growth of the patterned silicon surfaces. As is observed in figure 3 , the pyramid surface coverage and the pyramid sizes decreased with IPA concentration, which leads to about $71 \%$ loss in percent reflectivity compared to bare silicon, and a possible increase in light trapping as schematically shown in figure 2.

The measured percent reflectivity of the patterned silicon surfaces with different $\mathrm{KOH}$ concentrations of, 20, 24, 26 and $28 \mathrm{wt} \%$, in addition to $3 \mathrm{wt} \%$ of IPA solution at $80^{\circ} \mathrm{C}$ for $40 \mathrm{~min}$ is shown in figure 7 .

The behaviour of the surface specular percent reflectivity does obey the reasoning discussed earlier for the relationship between reflectivity and the pyramid sizes and the pyramid surface coverage. As the $\mathrm{KOH}$ concentration is increased, the pyramid size and surface coverage decrease. This behaviour is similar to what was observed for the case of IPA concentration. The percent reflectivity decreases by about $70 \%$ compared to bare silicon as the $\mathrm{KOH}$ concentration increases due to the loss of the pyramid structure as shown in figure 4 . The concentrations of both $\mathrm{KOH}$ and IPA are directly related 

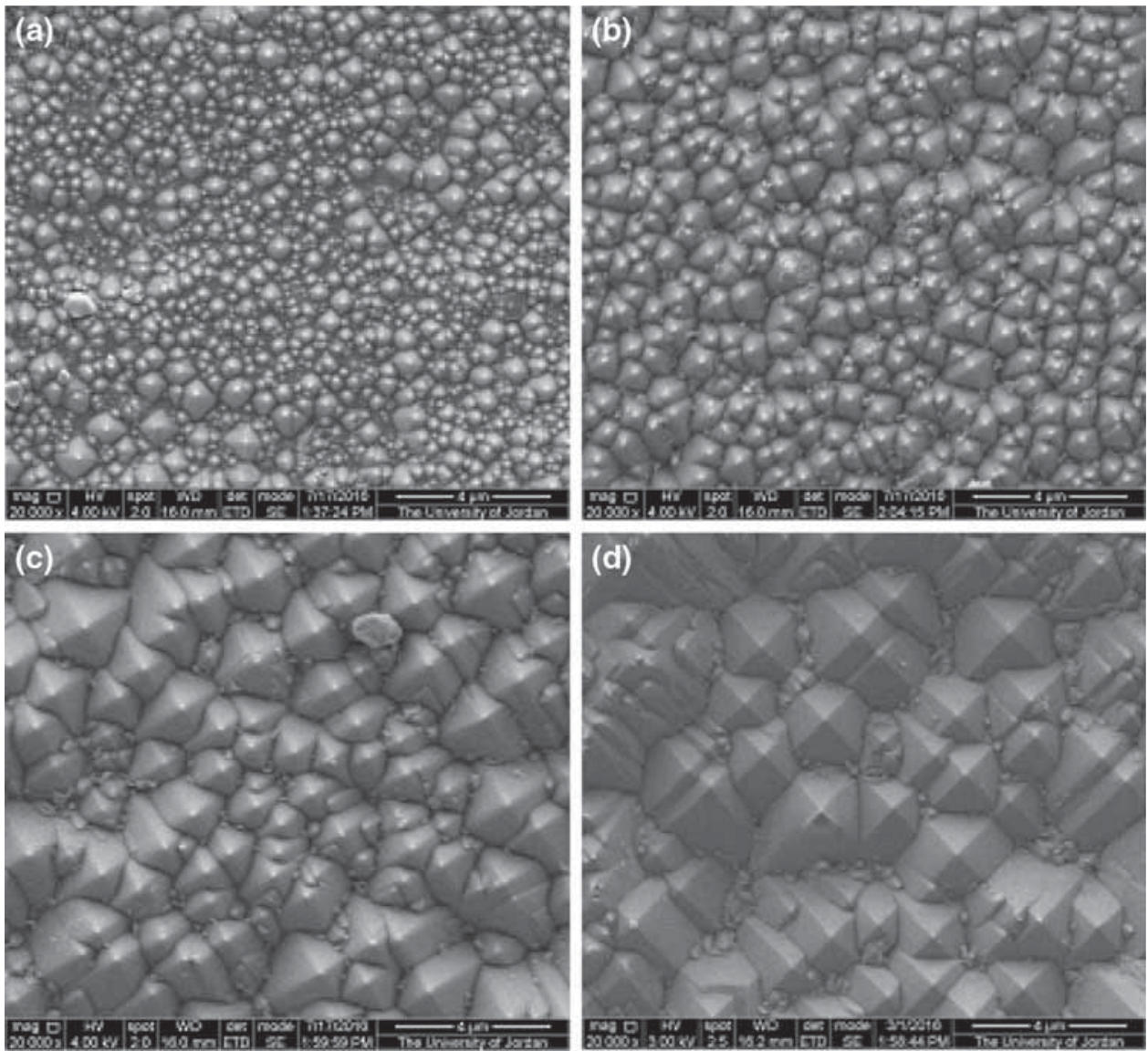

Figure 5. SEM images for silicon substrates after etching in a $20 \mathrm{wt} \% \mathrm{KOH}$ mixed with $3 \mathrm{wt} \%$ of IPA at $80^{\circ} \mathrm{C}$ for different etching times (a) 15, (b) 20, (c) 25 and (d) $30 \mathrm{~min}$.

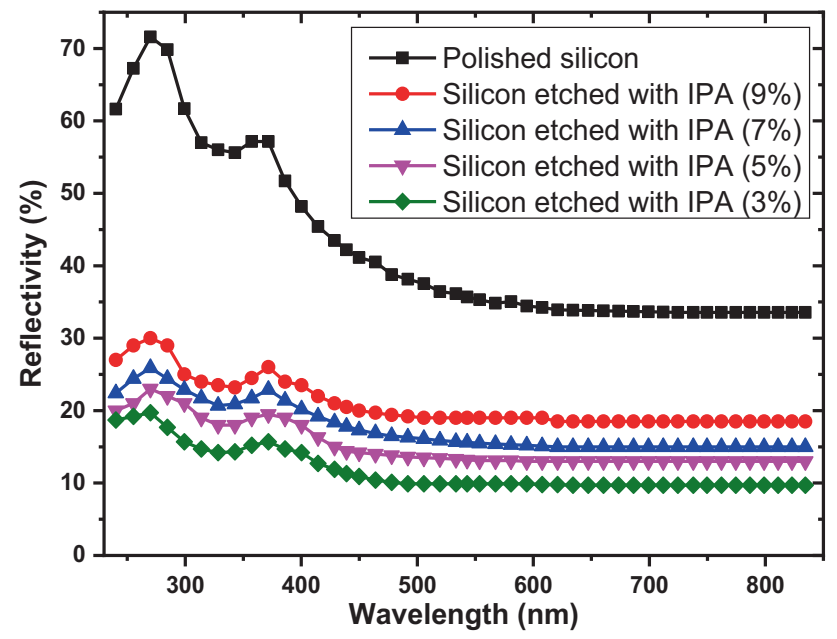

Figure 6. Reflection of polished and patterned silicon surfaces as a function of wavelength for samples etched at different concentrations of IPA.

to the silicon etching rates, which in turn controls the pyramid sizes and surface coverage [14].

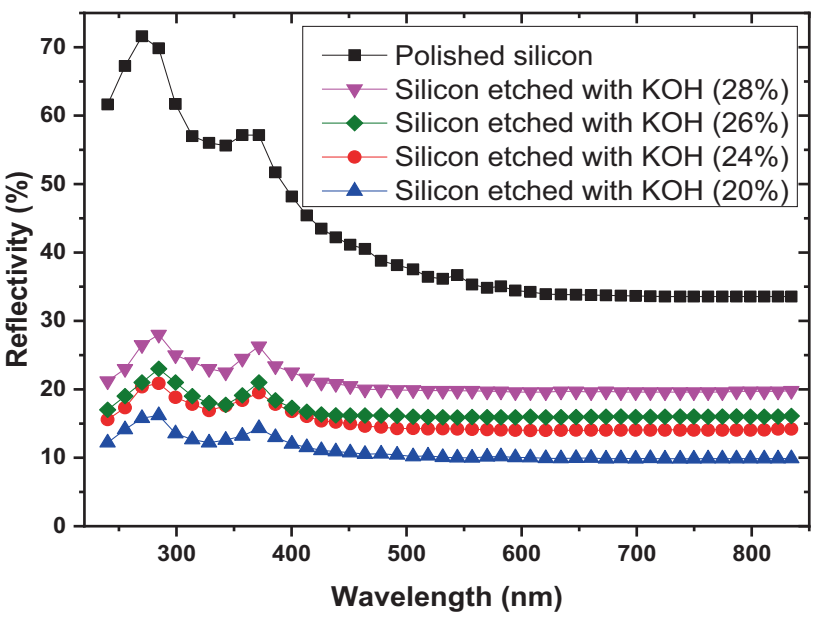

Figure 7. Percent reflectivity of the silicon surfaces as a function of wavelength for various $\mathrm{KOH}$ concentrations.

Figure 8 takes a closer look at the percent reflectivity at normal incidence of bare silicon substrates and substrates etched for 15, 20, 25 and $30 \mathrm{~min}$ in $20 \mathrm{wt} \% \mathrm{KOH}$ mixed 


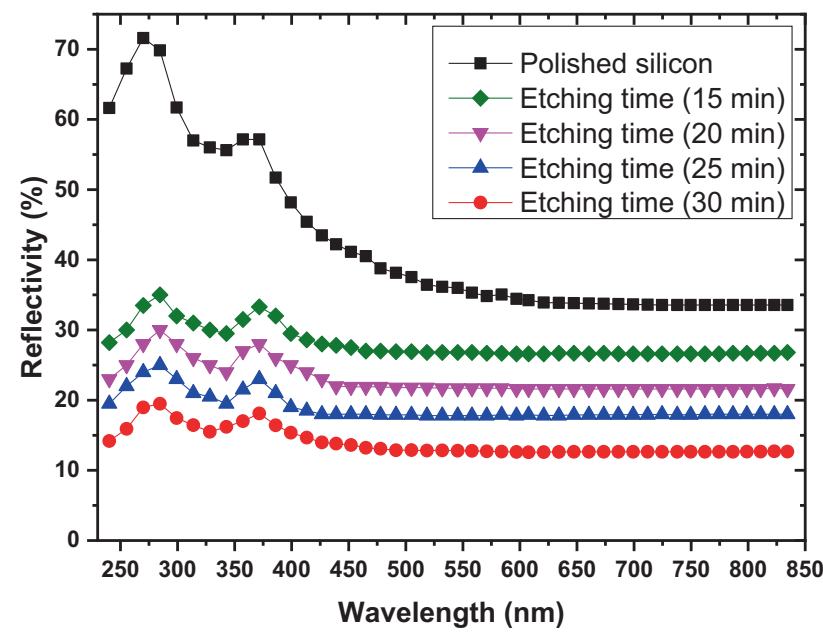

Figure 8. Percent reflectivity of silicon substrates $v s$. wavelength for various etching times.

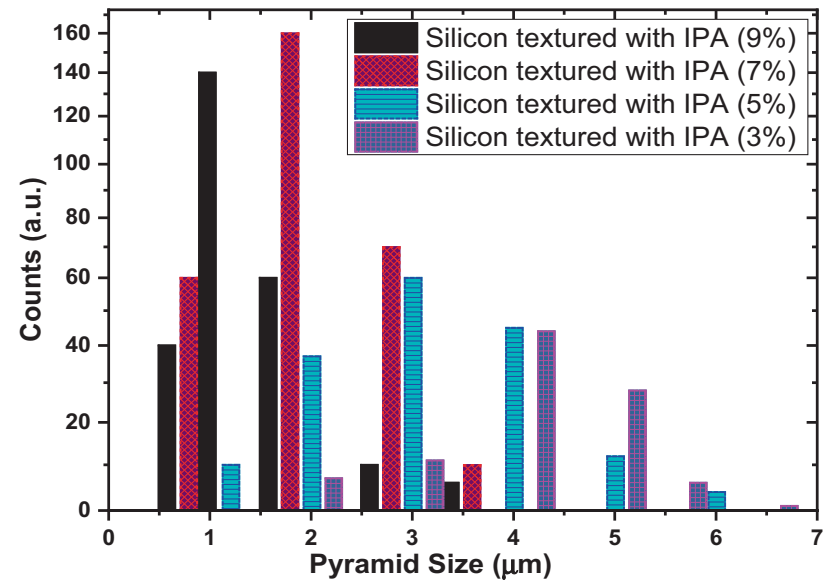

Figure 9. Pyramid size distribution obtained using Imagej software for various IPA concentrations.

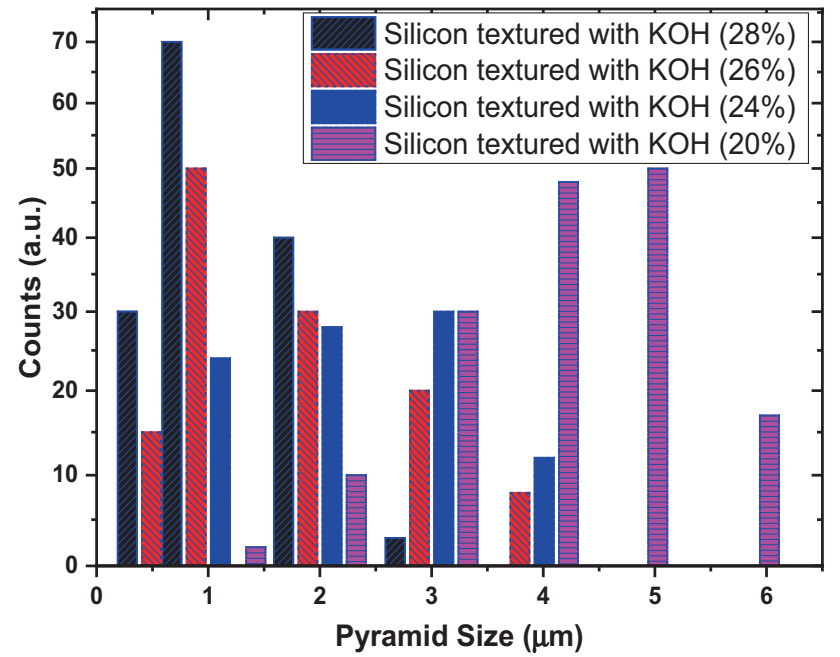

Figure 10. Pyramid size distribution obtained by using Imagej software for various $\mathrm{KOH}$ concentrations.

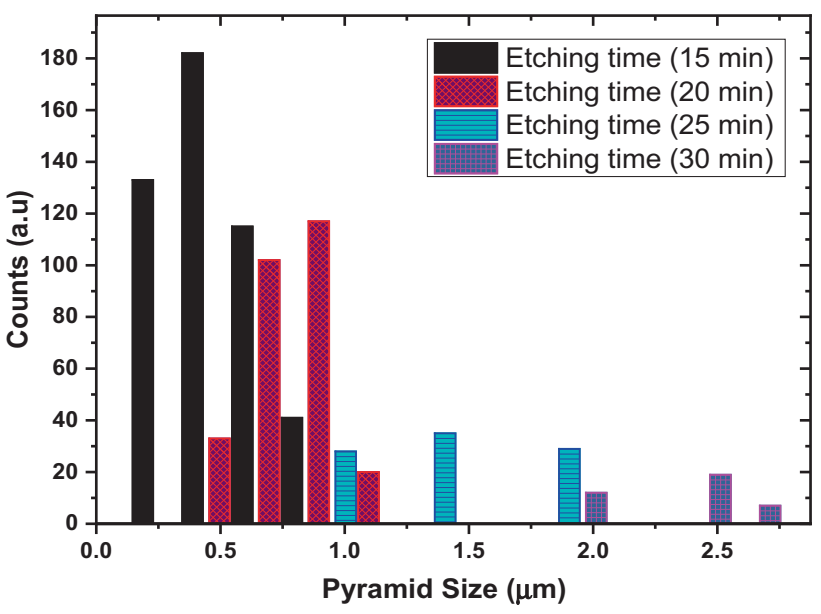

Figure 11. Pyramid size distribution obtained by using Imagej software for various texturization times.

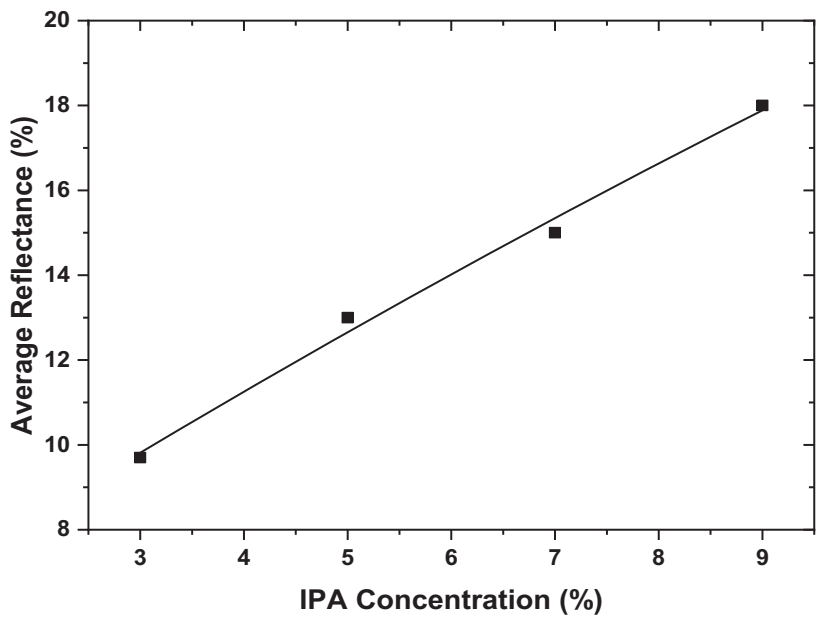

Figure 12. Percent reflectivity of etched silicon surfaces for different IPA concentrations at a wavelength of $650 \mathrm{~nm}$.

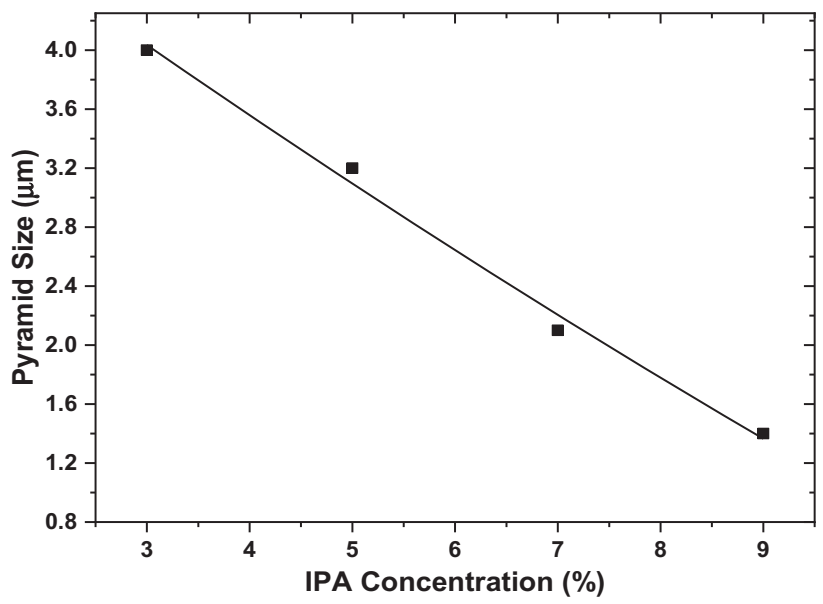

Figure 13. Pyramid size evolution for different IPA concentrations. 
with $3 \mathrm{wt} \%$ of IPA at $80^{\circ} \mathrm{C}$. The behaviour of the surface percent reflectivity is consistent with the earlier observations. As the etching time increased from 15 to $30 \mathrm{~min}$, the pyramids grew in size as shown in figure 5. Surfaces with larger pyramid sizes and a better surface coverage show lower percent reflectivity by about $63 \%$ compared to bare silicon and higher light trapping possibility. This behaviour follows the exact trend reported for the effects of $\mathrm{KOH}$ and IPA concentrations.

Figure 9 shows the distribution of pyramids and their sizes as a function of the IPA solution concentration. The largest pyramids were obtained at a concentration of $3 \mathrm{wt} \%$. The pyramid sizes reduced from $4.0 \pm 0.4$ to $1.4 \pm 0.3 \mu \mathrm{m}$ (mean $\pm \mathrm{sd}$ ), which correspond to height of 2.8 and $1.0 \mu \mathrm{m}$, respectively, as the IPA concentration increased from 3 to $9 \mathrm{wt} \%$. The reduction in pyramid sizes is attributed to the limiting reaction rates discussed earlier. The higher reaction rates allow the formation of a layer depleted of active etching agents that

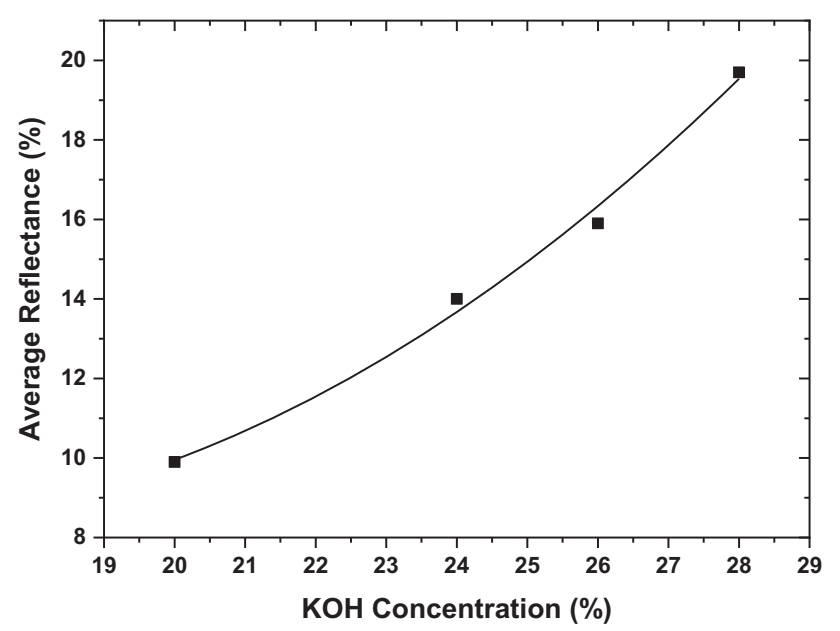

Figure 14. Percent reflectivity of silicon surfaces for different $\mathrm{KOH}$ concentrations for the wavelength of $650 \mathrm{~nm}$.

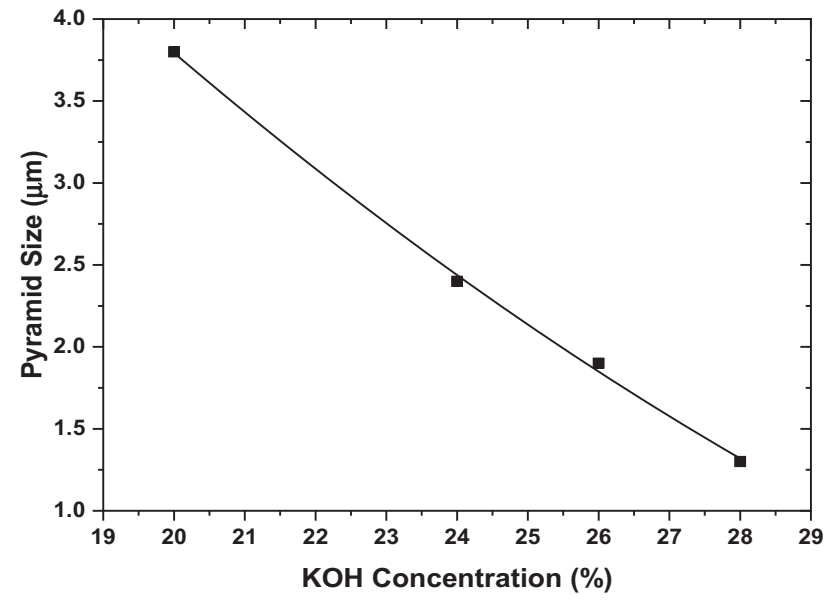

Figure 15. Pyramid size evolution for various $\mathrm{KOH}$ concentrations. lead to limited pyramid growth and hinder pyramid formation $[14,15]$.

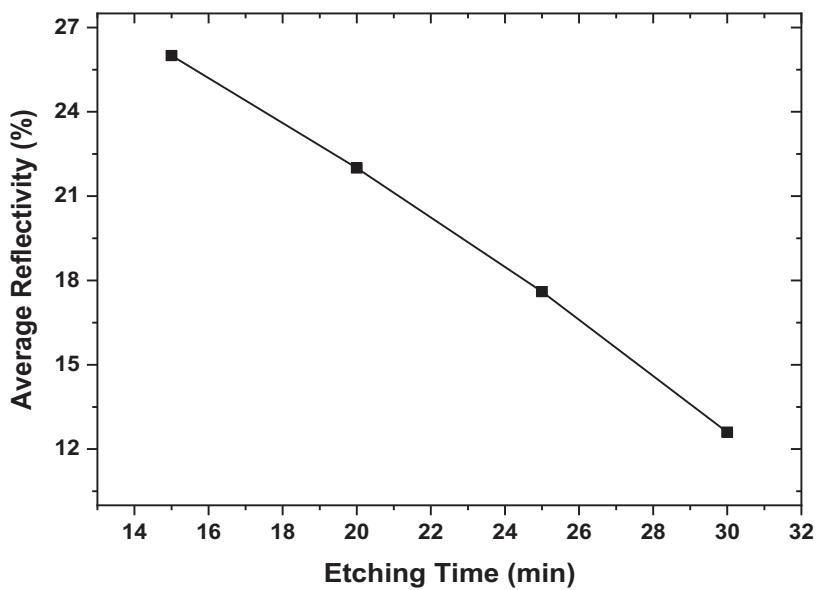

Figure 16. Percent reflectivity of Si substrates vs. etching time as measured at the wavelength of $650 \mathrm{~nm}$.

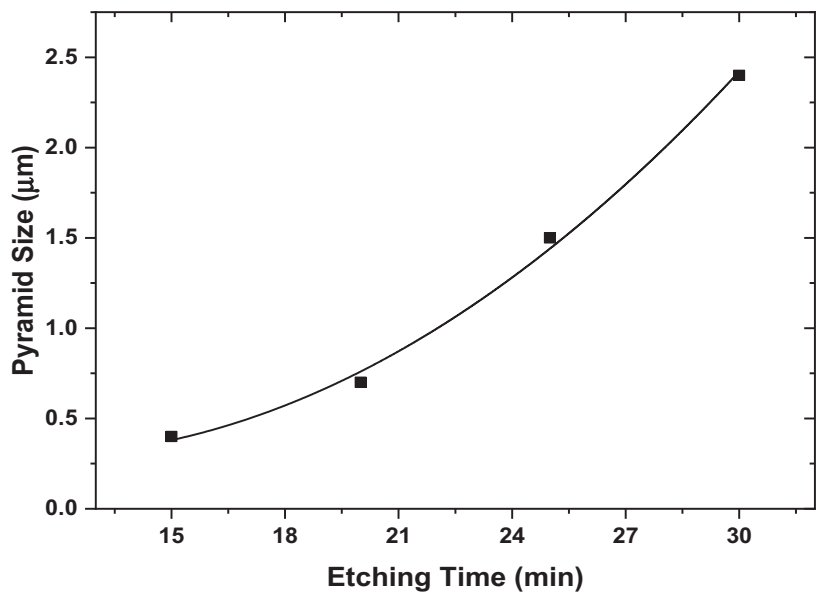

Figure 17. Pyramid size for various etching times.

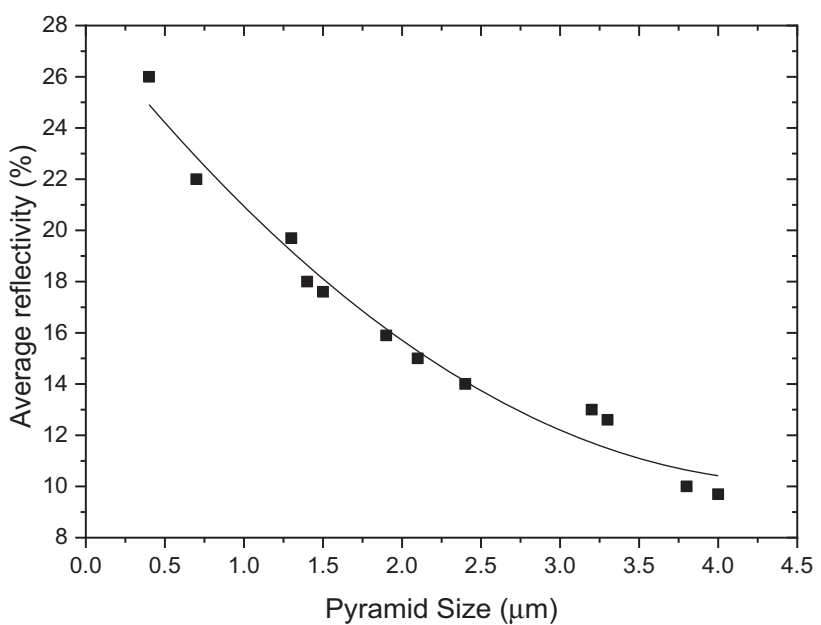

Figure 18. Percent reflectivity $v s$. average pyramid size. 
Table 1. Count of pyramids per $100 \mu \mathrm{m}^{2}$, pyramid size (mean $\pm \mathrm{sd}$ ), peak height (mean \pm error) and the average percent reflectivity for nine samples prepared at $80^{\circ} \mathrm{C}$ under different texturing conditions ( $\mathrm{KOH}$ concentration (wt $\left.\%\right)$, IPA concentration (wt\%) and etching time (min)).

\begin{tabular}{|c|c|c|c|c|c|c|}
\hline $\begin{array}{l}\text { Pyramid } \\
\text { group }\end{array}$ & $\begin{array}{l}\text { Texturing } \\
\text { conditions }\end{array}$ & Coverage $(\%)$ & Counts (a.u.) & $\begin{array}{l}\text { Mean pyramid } \\
\text { size }(\mu \mathrm{m})\end{array}$ & $\begin{array}{l}\text { Mean peak } \\
\text { height }(\mu \mathrm{m})\end{array}$ & $\begin{array}{l}\text { Average } \\
\text { reflectivity }(\%)\end{array}$ \\
\hline \multirow[t]{3}{*}{ Small } & $(20,3,15)$ & 79 & 488 & $0.40 \pm 0.01$ & $0.28 \pm 0.01$ & 26 \\
\hline & $(20,3,20)$ & 97 & 190 & $0.70 \pm 0.02$ & $0.50 \pm 0.02$ & 22 \\
\hline & $(28,3,40)$ & 82 & 44 & $1.3 \pm 0.1$ & $0.92 \pm 0.07$ & 19.7 \\
\hline \multirow[t]{3}{*}{ Medium } & $(26,3,40)$ & 93 & 26 & $1.9 \pm 0.1$ & $1.34 \pm 0.07$ & 15.9 \\
\hline & $(20,5,40)$ & 98 & 22 & $2.1 \pm 0.3$ & $1.49 \pm 0.21$ & 15 \\
\hline & $(24,3,40)$ & 98 & 17 & $2.4 \pm 0.3$ & $1.70 \pm 0.21$ & 14 \\
\hline \multirow[t]{3}{*}{ Large } & $(20,3,30)$ & 99 & 10 & $3.2 \pm 0.4$ & $2.26 \pm 0.28$ & 12.6 \\
\hline & $(20,3,40)$ & 99 & 7 & $3.8 \pm 0.2$ & $2.69 \pm 0.14$ & 9.9 \\
\hline & $(20,3,40)$ & 99 & 6 & $4.0 \pm 0.4$ & $2.83 \pm 0.28$ & 9.7 \\
\hline
\end{tabular}

Figure 10 shows the distribution of pyramids and their sizes as a function of $\mathrm{KOH}$ concentration. The largest pyramids were obtained at a concentration of $20 \mathrm{wt} \%$, and as the concentrations of $\mathrm{KOH}$ varied between 20 and $28 \%$, the size of the pyramids shrank from $3.8 \pm 0.2$ to $1.3 \pm 0.1 \mu \mathrm{m}$ (mean $\pm \mathrm{sd}$ ), which also corresponds to pyramid heights of 2.69 and $0.92 \mu \mathrm{m}$, respectively. As was mentioned earlier, the higher $\mathrm{KOH}$ concentrations lead to limiting reaction rates that prevent any further pyramid growth or pyramid formation $[14,15]$.

Figure 11 shows the distribution of pyramids and their sizes for various texturization times. The largest pyramid sizes were obtained for an etching time of $30 \mathrm{~min}$, and the size of the pyramids increased from $0.40 \pm 0.01$ to $2.4 \pm 0.04 \mu \mathrm{m}$ (mean $\pm \mathrm{sd}$ ), which also corresponds to heights of 0.28 and $1.70 \mu \mathrm{m}$, respectively, as the etching time increased from 15 to $30 \mathrm{~min}$. The longer growth times allow ample time for pyramid etching and growth. The changes in pyramid sizes and pyramid surface coverage have direct effects on surface reflectivity and light trapping properties as discussed earlier.

The effects of IPA concentration on the percent reflectivity at a wavelength of $650 \mathrm{~nm}$ are shown in figure 12. The percent reflectivity increases from $9.7 \%$, for the samples etched with $3 \mathrm{wt} \%$ IPA solution, to $18.7 \mathrm{wt} \%$ for substrates etched with $9 \mathrm{wt} \%$ IPA solutions. The increased reflectivity is indicative of the loss of the pyramid structure and a smoother and more regular surface of the processed substrates as presented in figure 13. Etching kinetics and the formation of the near surface layer effectively prevented IPA from reaching the surface and stopped any further etching of the Si substrate surface.

The percent reflectivity increases from 10 to about $19.8 \%$ as the $\mathrm{KOH}$ concentration changes from 20 to $28 \%$ for values measured at the wavelength of $650 \mathrm{~nm}$ as shown in figure 14 . Increased reflectivity is indicative of the loss of the pyramid structure and a smoother, more regular surface of the processed substrates as shown in figure 15 . The etching dynamics and the development of the protective layer are the main factors for the observed behaviour as previously mentioned. On the other hand, it is clear that the percent reflectivity increases with increased $\mathrm{KOH}$ concentration. These results of reflectivity measurements are consistent with the observed SEM results. The differences in the percent reflectivity are due to changes of surface roughness of the etched samples.

The percent reflectivity of the textured silicon surface decreased from about $25.2 \%$, for samples etched for $15 \mathrm{~min}$, to about $12.6 \%$ for samples etched for $30 \mathrm{~min}$ at a wavelength of $650 \mathrm{~nm}$ as shown in figure 16. This percent reflectivity behaviour reflects the fact that the pyramids are growing in size as the etching time is increased and this, in turn, reduces the normally reflected light across the whole studied wavelength range. The pyramid size growth as a function of pyramid etching time is presented in figure 17.

Statistical analysis of the SEM images was used to study the effects of pyramid size on the percent reflectivity of textured silicon surfaces. The results of the statistical analysis are presented in figure 18. It is clear that samples of smaller pyramid sizes $(0.4$ to $1.3 \mu \mathrm{m})$ have higher percent reflectivity than those of larger pyramid sizes $(3.2$ to $4.0 \mu \mathrm{m})$. For a detailed comparison and as a short summary, the statistical results on the different pyramid sizes studied in this work and their relation to the resulting percent reflectivity are presented in table 1. The presence of small pyramids and/or large pyramids does affect the light trapping behaviour of the textured Si surfaces. A clear decrease in percent reflectivity with increasing pyramid size, i.e., more light trapping, was also reported elsewhere [13,14]. As mentioned earlier, smaller pyramids show more horizontal facets and this leads to a higher percentage of reflected light [29]. Therefore, reflection losses were found to decrease with the appearance of larger pyramids.

\section{Conclusions}

The impact of $\mathrm{KOH}$ solution concentration on the production of the homogeneous pyramid pattern with low reflectivity 
values was investigated. But it is not just the $\mathrm{KOH}$ concentration that considerably influences the pyramid formation on Si substrates, but also some other factors such as etching time, IPA concentration and temperature. Therefore, all etching parameters have to be carefully investigated to obtain the desired optimal pyramid texture and thus to reduce the total reflection of silicon wafers. An inverse relationship was verified for the dependence of the percent reflectivity on the pyramid size. It was also found that the concentration of IPA is crucial for the formation of the pyramids and for their size distribution. It turned out that the IPA concentration is the limiting factor that controls the etching rate. The optimum etching solution determined by this work was a solution of $20 \mathrm{wt} \% \mathrm{KOH}$ with $3 \mathrm{wt} \%$ IPA for wet etching at a reaction temperature of $80^{\circ} \mathrm{C}$ with an etching time of $40 \mathrm{~min}$. With these optimal etching conditions and with a pyramid size of $4 \mu \mathrm{m}$, the percent reflectivity of the etched surfaces was measured to be around $9.7 \%$ for the wavelength of $650 \mathrm{~nm}$. This relatively low reflectivity value can be very useful for photovoltaic and solar cell applications.

\section{References}

[1] Svetoslav K, Martin S B and Martin S 2006 Appl. Phys. Lett. $\mathbf{8 8} 203107$

[2] Gangopadhyay U, Kim K, Dhungel S K, Basu P K and Yi J 2006 Renew. Energy 311906

[3] Sparber W, Schultz O, Biro D, Emanuel G, Preu R, Poddey A et al 2003 Proceedings of 3rd World Conference on Photovoltaic Energy Conversion (Osaka, Japan) p 1372

[4] Deng T, Chen J, Wu C N and Liu Z W 2013 ECS J. Solid State Sci. Technol. 2419

[5] Xiao J, Wang L, Li X, Pi X and Yang D 2010 Appl. Surf. Sci. 257472

[6] Rola K, Ptasinski K, Zakrzewski A and Zubel I 2014 Microsyst. Technol. 20221

[7] Indermun S, Luttge R, Choonara Y E, Kumar P, du Toit L C, Modi G et al 2014 J. Control. Release 185130

[8] Herwik S, Kisban S, Aarts A, Seidl K, Girardeau G, Benchenane K et al 2009 J. Micromech. Microeng. 19074008
[9] Yan L, Arnab D, Ziyin L, Ian B C, Ajeet R I and Wong C P 2014 Nano Energy 3127

[10] Sievert W, Zimmermann K U, Hartmann B, Klimm C, Jacob K and Angermann H 2009 Solid State Phenomena 145 223

[11] Baker-Finch S and McIntosh K 2011 Prog. Photovolt. Res. Appl. 19406

[12] Hongjie L, Honglie S, Ye J, Chao G, Han Z and Jiren Y 2012 Appl. Surf. Sci. 2585451

[13] Vazsonyi E, De Clercq K, Einhaus R, Van Kerschaver E, Said K, Poortmans J et al 1999 Energy Mater. Sol. Cells 57 179

[14] Jose N X 2013 PhD Thesis (Faculty of Sciences, Department of Physics, Konstanz)

[15] Keith R M and Luke P J 2009 J. Appl. Phys. 105124520

[16] Park S H, Park J, You K H, Shin H C and Kim H O 2012 J. Occup. Health 55120

[17] Zubel I and Kramkowska M 2001 Sens. Actuators A Phys. 93 138

[18] Drago R, Vrtacnik D, Aljancic U and Amon S 2003 J. Micromech. Microeng. 1326

[19] Tiago S M, Pamakstys K, Luis M G, Graça M and Susana C 2015 Micromachines 61534

[20] Yaqin W, Ruizhi L, Junjun M and Shi-Qing M 2015 5th International Conference on Advanced Engineering Materials and Technology

[21] Jinsu Y, Junsik C, Kyumin H et al 2012 J. Korean Phys. Soc. 602071

[22] Park H, Kwon S, Lee J S et al 2009 Sol. Energy Mater. Sol. Cells 931773

[23] Charanpreet S, Vijay K, Kiran W and Sood S C 2012 Int. J. Comput. Sci. Commun. Tech. 5974

[24] Sarro P M, Bride D, Vlist W V and Bride S 2000 Sens. Actuators 85340

[25] Singh P K, Kumar R, Lal M, Singh S N and Das B K 2001 Energy Mater. Sol. Cells 70103

[26] Kyu M H and Jin S 2014 J. Korean Phys. Soc. 641132

[27] Al-Husseini A M and Lahlouh B 2017 J. Appl. Sci. 17 1812

[28] Krzysztof P R and Zubel I 2013 Microsyst. Technol. 19635

[29] Jan K, Heike A, Uta S and Bert S 2013 Energy Proc. 38 833 